\title{
Design of a Tea Garden Antifreezing Control System
}

\author{
Biqing $\mathrm{Li}^{1, \mathrm{a}}$, Yan $\mathrm{Li}^{{ }^{*}, \text { a }}$, Chongjun Yang ${ }^{1, \mathrm{a}}$, Shiyong Zheng ${ }^{2, \mathrm{~b}}$ \\ ${ }^{1}$ College of Mechanical and Electronic Engineering, Hezhou university, Hezhou Guangxi \\ 542899, China; \\ ${ }^{2}$ College of Computer Science and Information Engineering, Hezhou university, Hezhou \\ Guangxi 542899, China; \\ ajanliful@163.com, b229292710@qq.com
}

Corresponding Author: Yan Li

Keywords: ZigBee, MPS430G2553, the upper and lower bound, DS18B20.

\begin{abstract}
The design is based on ZigBee network communication module, which is mainly composed of power supply, master control, display, temperature acquisition, key control section, howler alarm and so on. It mainly uses ZigBee network to monitor the temperature value of collection point. The temperature is collected from DS18B20, and then transmitted to the main control port through the ZigBee network. Set up control system with MPS430G2553 mixed signal microcontrollers to establish control systems. The display screen is able to display the real-time corresponding temperature of various monitoring points. The warning temperatures of different monitory point can be set individually. When the temperature exceeds the upper and lower bound, it gives an alarm automatically, and there is audible alarm at live monitoring point, also monitory points can be added according to personal needs. Suitable site can be selected to place mobile monitory point, the data transmission defense will be more secure and reliable. It uses batteries for power supply, and the operation is much more convenient, avoiding cumbersome stay wire and other operations.
\end{abstract}

\section{Introduction}

The design mainly uses Zigbee to realize wireless communication, selecting mixed signal microcontrollers MSP430G2553 as a means of control, using DS18B20 digital temperature sensor as the temperature acquisition part, together with the part of 12864 crystal display to display temperature, in combination with some components to achieve specific functions.

ZigBee module II, power supply and buttons, temperature and humidity sensor, motor and display, the master control communication module, ZigBee communication module acquisition control terminal, the liquid crystal display and buzzer. 


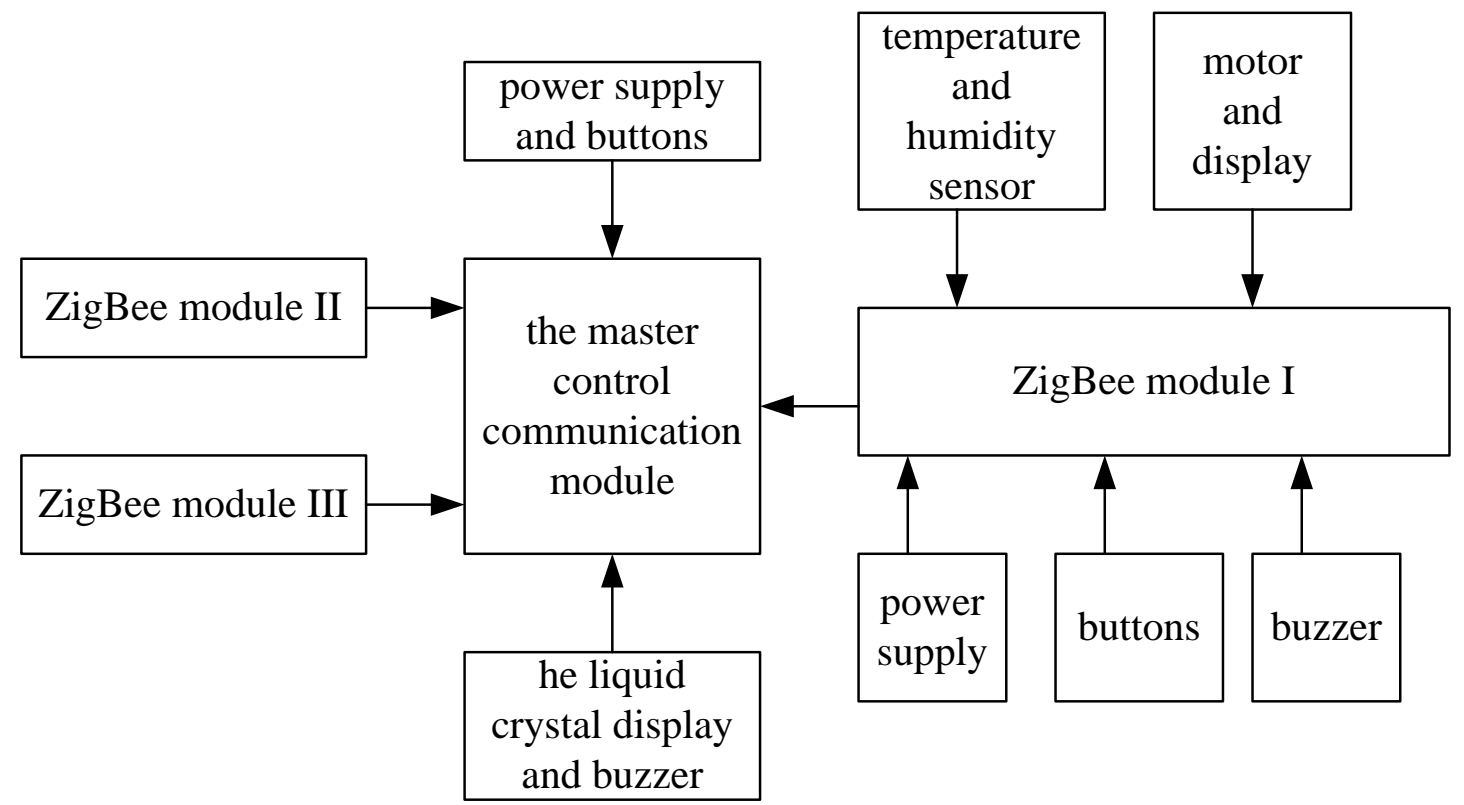

Figure 1 the System Diagram

The design is divided into master control module and acquisition module, with the main module controlling the whole work. Besides ZigBee wireless communication module, there is MPS430G2553 mixed microcontroller, and 12864 display screen can show the data of acquisition module. Buzzer will give alarm when the detected temperature exceeds the defined range.

Slave module is also known as acquisition module, and the collector is DS18B20, the temperature collected is transmitted to the main module for display through ZigBee wireless communication modules, and its own display will show the temperature of its location the first time, if the temperature exceeds the range, buzzer will ring. Through controlling the reflection of the control port, the switch of the motor can be started automatically or manually, thereby performing the freezing instructions.

\section{Hardware Design}

According to the functions involved, the principle of the design is mainly composed of master control circuit, ZigBee wireless communication circuit, temperature acquisition circuit, the howler alarm buzzer circuit, OLED display circuit, electric circuit and keying circuit, the principle of circuits at different part is as follows

\section{1 master control circuit}

In this design, the master control part adopts MPS430G2553 mixed micro-control system. Add reset circuit into the control circuit, when the system program can not work properly not because of the damage of components, press the reset button to restart the system, which is the equivalent of making the entire system running from the beginning of the program.

\subsection{Temperature Acquisition Circuit}

This design adopts single-bus temperature sensor DS18B20, the hardware circuit of which is relatively simple to use, and the reading of the temperature value is quite accurate, which is suitable for the temperature acquisition in general environment.

\subsection{ZigBee circuit}

In order to achieve the goal of free transmission of information between between wireless nodes, the ways of connection has gradually developed from basic series mode to the network mesh connection. Because of the intellectualization of each node in the mesh connection mode, when there is fault in any node in the network, the nearby wireless node will replace the fault node for the transmission and forwarding of information, thereby greatly improving the system reliability . 


\section{Conclusion}

The design is divided into host module and slave module, and the slave module display the sensed temperatureon the LED LCD through DS18B20 sensor, and the temperature range can be set as desired, to make the gap between the detected temperature and the temperature set on their own between $\pm 3{ }^{\circ} \mathrm{C}$, beyond which sound-light alarm will be made, at the same time, LED simulation heating and DC-fan can be used to simulate the up and down of temperature.

This work is supported the following fund :

2016 The project of improving the basic ability of young teachers in Colleges and universities in Guangxi:"Design and development of electronic commerce platform of agricultural products based on Semantic Technology”(No,KY2016YB455).

2016 Guangxi higher education teaching reform project: "Exploration on the cultivation mechanism of the teaching characteristics of Communication Engineering Specialty Based on ZTE ICT education platform”.

2015 college students' innovative training program: "Research on the application of value added travel experience in the mobile terminal of the 'ethnic custom travel' in Guangxi" (No 201511838070);\&\& “The design and development HeYuanTong Campus Mobile Phone APP based on Android” (No 201511838034).

2015 Teaching case project construction project of hezhou university: "Tourist positioning and guiding system project of Huangyao scenic";\&\& "Development and application of the information management system of 'Huang Yao Tong'”.

Nanling corridor of ethnic culture research base of Open Fund Project: "Nanling National corridor costumes digital display design” (No 2015kf27);

Project of scientific research and technology development project of Hezhou: "Design and implementation of agricultural products e-commerce platform based on Semantic Technology" (No,Hekeneng 1506006)

Scientific research project of hezhou university: "The intelligent home system Implementation Research based on the Internet of Things technology" (No 2014YBZK10); "Research and development of E-government platform based on mobile terminal” (No 2015ZZZK03);

Reform in Education project of hezhou university: "Internet of things"(No hzxytszy201501); "Research on application personnel training model of Communications for SMEs"(No hzxyjg201525);

Master degree discipline construction scientific research and Cultivation Project: "Research on the social work service of the teenagers' Network Addiction"(No 2015SHGZ005) \&\&"Research on the intelligent development of rural tourism in Guangxi based on the Internet of things" (No 2015MTA16);

\section{Reference:}

[1] Zidong Yang. Design of farmland straw straw burning wireless monitoring system based on ZigBee [J]. Agricultural Mechanization Research, 2009.

[2] Na Pang, Defu Cheng. Greenhouse monitoring system Design based on ZigBee wireless sensor network [J]. Jilin University Journal (Information Science Edition), 2010.

[3] Hanping Huang. Intelligent temperature measurement system design and simulation [J] 2012, (10): 255 -257. 